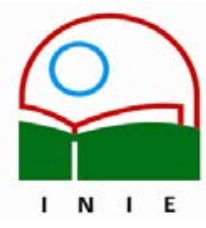

Universidad de Costa Rica

Facultad de Educación

Instituto de Investigación en Educación

ACTUALIDADES INVESTIGATIVAS EN EDUCACION

\title{
¿QUÉ NOS PUEDE APORTAR EL PSICOANÁLISIS EN LA COMPRENSIÓN DE LAS RELACIONES EN LA ESCUELA?
}

\author{
Kathia Alvarado Calderón ${ }^{1}$
}

\begin{abstract}
El gran interés de la pedagogía por el psicoanálisis, descansa en una tesis que se ha vuelto evidente. Sólo puede ser educador quien es capaz de comprenderse por empatía con el alma infantil (...) Freud, S. (1913-14/1997, p. 250)
\end{abstract}

Resumen: Este artículo se propone como resultado de la práctica educativa de la autora en ambientes escolares y universitarios. La necesidad de una formación sólida en teoría pedagógica a los estudiantes que inician su formación en Educación es importante, pero también lo es brindarles herramientas para enfrentar las demandas que los ideales educativos culturales imponen tanto a los educadores y educadoras como a los niños, niñas y adolescentes. La propuesta aquí esbozada es una reflexión teórica acerca de la pertinencia del concepto relaciones transferenciales, importado de la teoría psicoanalítica para la práctica pedagógica. El tema de la relación del psicoanálisis y la educación es retomado a lo largo de este ensayo.

Palabras clave: PSICOANÁLISIS Y EDUCACIÓN/ RELACIONES TRANSFERENCIALES/ INTERACCIÓN DOCENTE-ALUMNO/

Abstract: This article is intended as a result of the author's educational practice with students from School, High School and University. The need of a solid background in pedagogic theory is as important as the ability to face the demands impose by the cultural-educative ideals. This article is a theoretical proposal about the relevance of the transference concept, for the pedagogic practice. The subject of the education-psychoanalysis relationship is developed along the essay.

Keywords: PSYCHOANALYSIS AND EDUCATION/ TRANSFERENCE CONCEPT/ TEACHER-STUDENT INTERACTION/

\section{Introducción}

La escuela como sistema de relaciones, se estructura alrededor de tres elementos que la componen, a saber, quien enseña, los estudiantes y el saber enseñado, siendo anudados por el interés primordial de lograr el aprendizaje. Elementos que interactúan entre

\footnotetext{
${ }^{1}$ Licenciada en Psicología. Bachiller en Enseñanza de la Psicología. Investigadora del Instituto de Investigación en Educación (INIE). Psicóloga Educativa en el Centro de Asesoría Estudiantil de la Universidad de Costa Rica.
}

Correo electrónico: kathiaa@cariari.ucr.ac.cr

Artículo recibido: 21 de julio, 2004

Aprobado: 21 de febrero, 2005 
sí generando relaciones de diversa naturaleza según el objeto de la relación. Así tenemos que entre educador ${ }^{2}$ y saber ocurre una relación didáctica pues el principal objetivo es hacer trasmisible ese conocimiento. La relación entre aquel que aprende y el saber la conocemos como relación de aprendizaje, y la relación entre quien enseña y quien aprende, podemos considerarla como una relación propiamente dicha (Chevallard, Y. 2000).

La interacción en la escuela es para nosotros el despliegue de una serie de procesos afectivos, tanto entre camaradas como entre estudiantes y educadores. Esta dimensión afectiva queremos abordarla siguiendo algunos de los elementos aportados por psicoanalistas interesados en la relación psicoanálisis y educación. Dicho de otra manera, nos interesa tomar en cuenta al inconsciente en la relación pedagógica.

Lo afectivo en la teoría psicoanalítica ocupa un lugar muy importante. Por ejemplo, en el origen del síntoma encontramos que el afecto y la representación (acto de pensamiento) relacionados con un evento particularmente intenso, sufren destinos diferentes durante la represión y es sólo cuando estos dos elementos se unen de nuevo cuando podemos obtener el resultado eficaz del tratamiento analítico ${ }^{3}$.

Nuestra reflexión se refiere a los elementos afectivos, en tanto inconscientes, que se ponen en marcha al lado de los actos de pensamiento en toda relación humana, y de cómo esta relación podría incidir en la relación pedagógica.

\section{La Educación: ¿Un oficio imposible?}

Desde la publicación de la Interpretación de los Sueños en 1900, la expansión del Psicoanálisis ha influenciado muchos campos de conocimiento. La Educación no es la excepción, Freud mismo dedicó algunas reflexiones a la relación entre psicoanálisis y

\footnotetext{
${ }^{2}$ Apreciamos el uso de la distinción genérica en las palabras, sin embargo, para facilitar la lectura de este texto, recurriremos a los términos educador o niño comprendiéndose al mismo tiempo educadora y niña. Extiéndase esta consideración cuando mencionamos el término sujeto (concepto importante en la teoría psicoanalítica) en el sentido femenino y masculino.

${ }^{3}$ Una referencia que recomendamos para quien le interese profundizar sobre este tema es el trabajo de A. Green conocido en francés bajo el nombre de L'affect publicado en 1999, sirva esta mención para subrayar el papel del afecto para la subjetividad.
} 
educación e incluso se le recuerda por una frase de sus escritos en "Análisis terminable e interminable" (1937/1981), donde nos ilustra la envergadura del propósito de educar; parafraseándolo, psicoanálisis, educación y política tienen al menos esto en común, que son tres oficios imposibles.

¿En qué radica la imposibilidad de la Educación? Pues bien, la imposibilidad de Educar consiste en el mantenimiento de una Educación definida según sus fines y propósitos como una acción ejercida por un adulto sobre un ser joven, con miras a su desarrollo físico, intelectual y moral y a su integración en el medio en el cual está destinado a vivir.

La imposibilidad radica en sostener que la Educación puede conducir al individuo a la feliz expansión de éste con miras a su perfección, a su felicidad y a su destino social. Ejerciéndose sobre el niño y la niña desde su nacimiento, con el propósito de que pueda llevarse al infante de etapa en etapa hacia la autonomía del adulto, otorgándole la instrucción y la cultura (Lafón, 1963).

La construcción de un tal concepto de Educación resume en parte la herencia de otras nociones venida de una antropología tradicional donde los elementos religiosos se han difuminado con la intención de desarrollar una idea "espiritual", "humanista" pero con la misma exigencia disciplinaria, el control de los instintos y de las pasiones pulsionales para alcanzar lo propio de lo humano como ideal, la racionalidad (Sotelo, 1999).

Los elementos que definen esta Educación retoman una visión moderna de la "nueva concepción educativa" sobre la conquista de la libertad y de la autonomía del individuo asociado con su racionalidad. Pero aún para nuestros días, afirma Sotelo I. (1999), la educación como paideia, tal y como se entiende desde los orígenes griegos, precisa de un paradigma ideal del ser humano, en referencia a un sistema de valores. Por tanto, a pesar de la coexistencia de los distintos modelos educativos de nuestras sociedades, la construcción de la noción de Educación se constituirá a partir de su paradigma del ideal de ser humano.

Para nuestra reflexión es importante subrayar algunos aspectos que llaman nuestra atención. El primero de ellos es la presentación de un educador cuyo papel es central en el proceso de aprendizaje, conocemos bien lo fundamental que éste es en la relación didáctica entre sujeto que aprende y objeto de conocimiento, ocupando un papel protagónico en la 
formación del niño y la niña. Sin embargo, los y las estudiantes no son individuos pasivos en este proceso, afirmación que no es nueva para nosotros. Ellos llegan a nuestras aulas con un gran bagaje familiar, tanto de conocimiento como en sus modos de interacción.

El segundo aspecto tiene relación con la definición de Educación en tanto "acción”, en este sentido ella se hace objeto de una técnica particular, pero parecida a las demás técnicas en tanto supone en primer lugar un conjunto de conocimientos positivos que consideran, sea la materia (el niño, la niña), sea las condiciones del medio donde se ejerce su acción. Definida de esta manera, la Educación se encuentra entre la ciencia y la técnica, como técnica establece reglas prácticas, como logos, tiende a establecer leyes generales, científicas y racionales.

Pero como señalamos más arriba, la Educación se define también a partir de sus fines y esto es la referencia a un sistema axiológico que le conduce en su actuar haciéndola perder toda neutralidad objetiva. De esta manera la Educación se dirige a procurar la feliz expansión del niño y de la niña hasta conducirlo a la autonomía en la edad adulta, lograda por supuesto gracias a una acción directa de la enseñanza a través del comportamiento del educador.

Todo ello nos lleva además a detenernos en la relación entre educación y pedagogía para precisarla. Consideramos en términos generales que la pedagogía es la técnica de la ciencia de la educación. Este es el arte de la educación: la puesta en marcha por parte de cada educador de los conocimientos, de la doctrina y de los principios que la pedagogía con su base científica le enseña. Pero en tanto que la pedagogía como la educación se enfrenta con el problema de los fines, esto la hace diferenciarse de otras técnicas propias de las disciplinas científicas.

La pedagogía se inscribe en la preocupación del deber ser, en esta medida se aleja de los fundamentos de una ciencia empírica. Así, la pedagogía considera no sólo los fundamentos científicos de otras disciplinas sino que además comprende una creación estética, una didáctica y una práctica filosófica que incluye la preocupación por la moral. 
Autores como Hubert (1965) y Lerbet (1980) han destacado esta complejidad con la cual el especialista se tropieza al respecto de la noción de pedagogía lo cual le conduce a conformarse con una definición que podríamos denominar semicientífica,

la pedagogía tiene por objeto elaborar una doctrina de la educación, a la vez teórica y práctica como la doctrina de la moral de la cual ella es la prolongación y que no es exclusivamente ni ciencia, ni técnica, ni filosofía ni arte, sino todo esto en conjunto y ordenado según articulaciones lógicas (...) La acción pedagógica está subordinada a la consideración de sus fines, lo que no es asunto de la ciencia, ni de la técnica, sino de consciencia y por consecuencia, de filosofía (Lerbet, 1980, p. 14)

El último aspecto que queremos mencionar es que la educación, en un sentido amplio, abarca los ámbitos de la instrucción escolar pero también la formación cultural del individuo, para lo cual éste debe adquirir hábitos y desarrollar habilidades que le permitan adaptarse a su medio social. La educación, es entonces entendida como la mediación para la manifestación de las facultades y potencialidades que cada individuo tiene en su ser personal (Dengo, 1998). Significa esto que la educación no se define solo como una acción sino el resultado de esta acción que da hábitos intelectuales o manuales y que por otro lado aporta cualidades morales.

La Educación procura que el niño y la niña se acerquen a la mayor perfección humana posible en el estado adulto. Pero este ideal, conducir a niños y niñas a la libertad de la autonomía en su adultez, por mediación de la acción pedagógica, supone un sujeto cuya naturaleza pulsional debe ser dominada para acceder a la cultura. Un niño cuya naturaleza primitiva debe ser moldeada por el adulto para que pueda convivir en su medio social.

La acción del educador se propone como fundamento del proceso, quien posibilita que el niño desarrolle sus capacidades y habilidades para ser parte de su grupo social. Un educador cuya mediación se efectúa entre la naturaleza primitiva y la esencia de lo cultural. Pero en este intento, el niño de la realidad parece haber sido olvidado en la abstracción del ideal educativo.

Asimismo, no podemos ignorar que al proponer la acción de la educación a partir de la participación del educador, hace imposible pensar en un proceso educativo 
descontextualizado de las realidades particulares que vivencian sus actores como Sujetos ${ }^{4}$.

El educador integra el par educador-educado como una alteridad, en una escena de relación donde el primero permite la expansión de las potencialidades que están contenidas en el sujeto. Pero pensar que el educador es un mero "factor" de la situación educativa, nos hace descuidar la consideración de los aspectos subjetivos e inconscientes inherentes a la relación pedagógica. Esto nos conduce a la vez a creer que es posible realizar el ideal de la técnica y de las reglas generales de base científica en dicha relación, como una pedagogía objetivista centrada en criterios cientificistas.

Lerbet, G. (1980) subraya que, definir qué es pedagogía tomando como base a las ciencias positivas, nos conduce a la objetivación, según un modelo fisicalista, de aquel que aprende, caracterizándose por el acento en la tercera persona (impersonal). En cambio, poner el acento en el "arte del educador" o sobre el contenido de su discurso (su saber), corresponde a una centralización sobre el polo magistral o sobre el educador; transformándose en una pedagogía que funciona en primera persona. Esto ha dado origen, afirma el autor, a diferentes corrientes que llevan como apelativo pedagogía tradicional, o

"logotrópica" según Caselmann, "centrada sobre el programa" según J.C. Filloux (1966) o aún "magistrocentrista" según J. Vial (1971) (...) Por esto una visión distinta de la pedagogía tradicional tendría que considerar al "objeto relacional" de la pedagogía, lo que permite elaborar un modelo en segunda persona, es decir, centrada en la relación afectivo-social facilitadora, maestro-alumno (Lerbet, 1980, p.4)

Este enfoque pedagógico nos subraya la relevancia de lo afectivo-relacional en las situaciones de aprendizaje. Pero de acuerdo con lo expuesto hasta aquí y a la luz de la teoría psicoanalítica, asumir lo afectivo de las relaciones en la escuela, nos lleva a la redefinición de la práctica pedagógica misma.

\footnotetext{
${ }^{4}$ Para el psicoanalista R. Steichen (1997) existe una diferencia entre sujeto de la razón consciente y Sujeto del pensamiento inconsciente, como producto simbólico.
} 


\section{De la relación entre psicoanálisis y educación.}

Freud (1932-36/1979) afirmó, a propósito de la relación entre psicoanálisis y educación, que se trataba de algo "importantísimo, ofrece grandísimas esperanzas para el futuro, quizás es lo más importante de todo cuanto al análisis cultiva, me refiero a la aplicación del psicoanálisis a la pedagogía, la educación de la generación futura" (p.135)

Para comprender la relación entre psicoanálisis y educación ponemos de relieve tres conceptos básicos que a la luz de esta reflexión adquieren un valor especial en nuestro trabajo cotidiano en las aulas. Estos son, "lo infantil", "la cultura" y "el Sujeto". La cuestión acerca del "niño" obliga a pensar de nuevo la racionalidad psicopedagógica, introduciendo la reflexión acerca del inconsciente psicosexual. La cuestión sobre la "cultura" nos presenta a la neurosis como su síntoma, el cual es vivido como un cierto conflicto entre lo pulsional y lo cultural. Y la cuestión del "Sujeto", es la pregunta por aquel que va a constituirse por el deseo y ese sujeto que será fantaseado por el "ideal educativo" (Assoun, 1997).

Con Freud la conceptuación de la infancia se modifica completamente. Con la teoría de las pulsiones descubrimos una nueva dimensión en la comprensión de lo infantil. La teoría sexual infantil pone de relieve que cualquier propuesta que tome en cuenta al niño como su objeto debe considerar que ante todo el niño se enfrenta al conflicto entre la libre expansión de sus necesidades libidinales y las necesidades de integración a lo social que impone su grupo de referencia.

Pero el sometimiento de la pulsión al ideal cultural es el origen y la "cristalización" de síntomas neuróticos. Es el neurótico quien descubre el carácter ineducable de la pulsión, lo que pone precisamente en fracaso a tan ansiado ideal, que interroga a su vez al ideal educativo mismo. La educación se ve así en medio de la pulsión y la cultura. Por tanto, la Educación no puede conducirnos al ideal de un adulto autónomo, pues su misma inscripción en la cultura obliga al Sujeto a ser frustrado en su deseo. En ello radica la imposibilidad de educarlo para el ideal educativo.

En el texto Nuevas Conferencias de Psicoanálisis de 1932, Freud se pregunta por la pertinencia de que el niño inicie un análisis de carácter profiláctico para prevenir los trastornos adultos, lo que de partida supone una sociedad abierta al psicoanálisis. Para Freud (1932-36/1979) la tarea de la educación es que "el niño aprenda a dominar sus 
pulsiones", lo contrario, es decir la libertad sin restricciones, es imposible. En tono jocoso dirá que eso sería interesante para los psicólogos pero no para los padres ni para los propios niños. Las consecuencias se verían tarde o temprano en el desarrollo, (y continúa) reprimir e inhibir las pulsiones es precisamente lo que origina el peligro de una enfermedad neurótica.

Entonces, ¿cuál educación es posible? Freud nos dirá, la educación debe pues buscar su camino entre la Escila del laisser-faire y la Claribdis de la denegación (frustración) (...) es necesario encontrar un óptimo para la educación para que ella pueda cumplir el máximo y perjudicar el mínimo. Se trata de decir hasta dónde se puede prohibir, en qué momentos y por cuáles medios. Además es necesario considerar el hecho de que objetos de la influencia educadora aportan con ellos disposiciones muy diferentes (1932/1979, p.138).

Pero Freud no pierde de vista el par educador-educando y trae a la consideración de todos las exigencias y expectativas que son dirigidas al primero, a saber; reconocer las necesidades del niño, adivinar lo que ocurre en su vida psíquica, darle amor, enseñarle contenidos y además conservar al mismo tiempo una parte eficaz de autoridad. A lo que nosotros agregaremos, el número de estudiantes por clase, el volumen de material que deberá ser calificado fuera del horario de trabajo, y en muchísimas ocasiones, velar por el proceso educativo de los propios hijos.

Motivo por el cual Freud considera la importancia de una preparación apropiada para cumplir con el oficio de educador, es decir, un conocimiento de la teoría psicoanalítica, y un tutor que conociendo de psicoanálisis pueda apoyar y supervisar su trabajo. $Y$ en este sentido sería mejor si el educador ha realizado un análisis personal, pues sin experiencia sobre su propia persona no podría apropiarse de la teoría analítica.

Freud asegura, "el análisis de maestros y educadores parece ser una medida profiláctica, más eficaz que la de los niños ellos mismos, pero también hay menos obstáculos que se oponen a su ejecución" (1932/1979, pp.139-140). He aquí la profilaxis de las patologías de la relación educativa, educar al educador en el "sentido de lo ineducable" (la pulsión). Principios que fueron retomados por muchos de los primeros psicoanalistas de los años de 1930 que trabajaron los fundamentos para una pedagogía psicoanalítica (Moll, J. 1989). 
De modo que la teoría psicoanalítica no solo evidencia la ilusión que contiene la teoría pedagógica al respecto de un discurso del Ideal infantil sino también a propósito del narcisismo herido del educador, quien durante su práctica retoma fuerza con la esperanza de un futuro mejor y el sueño de un niño forjado para el éxito (Mosconi, 1986).

Pero tampoco proponemos una sustitución del ideal pedagógico por una especie de ideal psicoanalítico, lo que además no sería posible, pues la teoría analítica devela claramente la constitución del sujeto por su falta (por su fractura narcisística). Con Freud presentamos una reflexión ética, es decir, ni ideales imaginarios personales, ni imaginarios sociales.

La Educación encuentra su significación como momento social de la constitución del Sujeto en su confrontación a un cierto orden simbólico (Assoun, 1997). De modo que una educación con orientación psicoanalítica no puede estar al servicio de un orden social sin considerar si es válido o defendible, por lo que Freud planteaba como necesario fijar otro objetivo más elevado para una educación inspirada por la teoría psicoanalítica como ciencia aplicada.

Para Freud no cabía duda que la educación con orientación psicoanalítica podía influir en la "salud" del sujeto, lo cual entendía en el contexto de una realización de éste que no lo ubicaría jamás del lado de la reacción (acting) ni de la represión. Para este autor, el psicoanálisis tiene un imperativo en relación con la confrontación del sujeto a un cierto "orden social" pues el psicoanálisis, según Freud, contenía suficientes factores revolucionarios para asegurar que lo que éste había develado no cambiaría.

En Freud, encontramos que la solidaridad entre educación y psicoanálisis, consiste en aquello que no pueden, es decir, en la falta. Aclaramos, lo que crea esta afinidad entre "acto pedagógico" y "acto psicoanalítico" y con el "gobernante", como oficios imposibles, es el hecho de que esta trilogía de actores tienen inminentemente que "vérselas" con el deseo del otro. Educado, gobernado o analizado, estos quieren actuar sus pasiones y poner en regreso la pulsión de curar, de educar y de gobernar. Sin embargo a diferencia de gobernar, tanto curar como educar tienen que ver con un saber, si bien de una naturaleza distinta. 
De modo que la nueva concepción pedagógica inspirada en la teoría psicoanalítica permitiría que el educador pudiese ser educado por el niño (a) mismo, como Sujeto confrontado a las exigencias de la pulsión pero al mismo tiempo en el reconocimiento de la imposibilidad, esto es, aceptar las propias fracturas y las del otro.

\section{De la aplicación del psicoanálisis a la educación.}

En esta exposición seguimos a M. Cifali (1982) y a N. Mosconi (1986) quienes consideran que la aplicación del psicoanálisis a la educación es posible a condición de no transformar a la teoría psicoanalítica en una teoría "madre" en el sentido de paralizarla en certezas dogmáticas, en espera de ser confirmada o confrontada por la educación como campo de aplicación y validación. Una pretensión de este tipo conduciría al investigador al riesgo de ostentar en una posición narcisística e imaginaria en un saber pleno y sin falta.

Una teoría psicoanalítica aplicada al campo de la educación debe estar dispuesta no solo a hacer surgir en este campo "preguntas inéditas" por el reconocimiento de la presencia del inconsciente en la práctica educativa, sino que también deberá estar dispuesta a ser modificada por su objeto mismo.

En particular, la teoría deberá tomar en cuenta todo lo que distingue las prácticas educativas de la práctica de la cura en tanto que relación dual; aquellas son -en efecto-, prácticas de grupo, trátese de la educación un grupo familiar o institucional sustituto, o de prácticas de enseñanza en el grupo-clase o de la formación en el grupo de formación. Una teorización de estos fenómenos supondrá, entonces, un llamado a los investigadores que procuran elucidar desde un punto de vista psicoanalítico todo lo que se desprende de los fenómenos de grupo (Mosconi, 1986, p.77)

En lo que concierne específicamente al trabajo en aula (grupo-clase), cuya finalidad es la enseñanza, subraya J, Filloux y desde antes M. Klein en sus primeros trabajos, que el grupo-clase presenta particularidades donde se pone en juego la problemática de la sublimación (cit. Por Mosconi, 1986.). Pero como señala Mosconi (1986, p.77), "solo los conocimientos en psicoanálisis de la educación podrán proporcionar a las prácticas educativas y educadores los datos que permiten constituir en este ámbito un saber práctico". Esto es, la aplicación del psicoanálisis a la educación requiere de una solidaridad entre 
ambas teorías de conocimiento, implicando que el investigador (a) conozca ambos campos de aplicación.

La pregunta sobre quién deberá poner en práctica esta aplicación tiene una respuesta formulada en paralelo con la práctica clínica, es decir, quien realiza la práctica educativa, puesto que hay una construcción personal y del objeto de conocimiento dentro del espacio educativo. Siendo fieles a las enseñanzas del psicoanálisis esto supone un Sujeto en reflexión en el sentido de quien gestiona personalmente un saber y una simbolización sobre sí mismo y su práctica, lo que le proporciona los elementos para poder descifrar e Interpretar aquello que surge en el encuentro con el otro del par educador-educando.

Esta propuesta implica un comprometerse consigo mismo y con lo avanzado por otros al respecto de la aplicación del psicoanálisis a la educación en la experiencia práctica. Posición teórico-reflexiva que permite un compromiso con una práctica educativa que reformula a su vez el ideal de la pedagogía tradicional porque el psicoanálisis devela las ilusiones y evidencia las fracturas (de este ideal).

\subsection{Las relaciones de transferencia.}

El psicoanálisis ha elaborado una gran serie de conceptos que podrían ser mencionados al considerar el proceso de aprendizaje. Sin embargo, ahora quisiéramos centrarnos en el concepto de transferencia. Pero antes, queremos subrayar que es necesario cuidar la rigurosidad teórica al importar cualquier concepto de un campo de conocimiento a otro, pues existe el riesgo de desvalorizar lo que éste significa.

El término relaciones de transferencia surge al teorizar un tipo de relaciones surgidas dentro del campo de la práctica clínica psicoanalítica. Para aclarar el concepto de transferencia recurrimos al Vocabulaire de la Psychanalyse (1998). En él Laplanche y Pontalis indican que se designa de esta manera al proceso por el cual los deseos inconscientes se actualizan sobre ciertos objetos en el marco de cierto tipo de relación establecida con ellos y eminentemente en el marco de la relación analítica. Y continúan, se trata de una repetición de prototipos infantiles vivida con un marcado sentimiento de actualidad. Esto para la cura analítica será muy importante pues su instalación, sus modalidades su interpretación y su resolución la caracterizarán. 
No obstante, la escuela proporciona un espacio muy importante de vinculación entre estudiantes y maestros (as), por lo que se hace posible aplicar el conocimiento que se ha desarrollado en esta área en el ámbito de las relaciones en la escuela. En todo caso, los procesos transferenciales no fueron una creación del psicoanálisis, sino que la situación clínica proporcionó las condiciones para su reconocimiento. De allí que pensemos en la viabilidad de la aplicación de este concepto en las relaciones de la escuela.

Ya desde los inicios del psicoanálisis Freud se refería a este aspecto en una conferencia que impartió bajo el nombre La psicología de los Colegiales en 1914. Para Freud el educador no sólo actúa en el proceso de aprendizaje, el educador además evoca en sus estudiantes la idealización primitiva con su propio padre, él observó,

(de estudiantes) transferimos sobre ellos el respeto y las expectativas del omnisciente padre de nuestros años infantiles (...) Les salimos al encuentro con la ambivalencia que habíamos adquirido en la familia, y con el auxilio de esta actitud combatimos con ellos como estábamos habituados a hacerlo con nuestro padre carnal. Si no tomáramos en cuenta lo que ocurre en la crianza de los niños y en la casa familiar, nuestro comportamiento hacia los maestros sería incomprensible, pero tampoco será disculpable. (Freud, 1913-14/1997, p. 250)

El educador ejerce su acción pedagógica en el contexto de una formación social y concretiza en sí, una red simbólica de representaciones asociadas con una autoridad (pedagógica), como una especie de relación arquetípica con las autoridades de la infancia.

Desde los primeros trabajos psicoanalíticos sobre lo infantil, se ha abordado de manera importante el impacto de otras figuras adultas, no parentales, para su vida afectiva. Los estudios e investigaciones de J. Bowlby (1989; Berk, L., 1999) muestran suficientemente la importancia de las primeras relaciones de los niños con sus cuidadores.

Por esta razón, nos interesa sobre manera cómo generar mejores condiciones en el salón de clase para el desarrollo de niños, niñas y adolescentes. Como señaló Freud (1937/1981), nuestro único interés, "es lograr las condiciones psicológicas mejores posibles para las funciones del yo, con esto (el psicoanálisis) ha cumplido su tarea". (p. 3362) 
Nuestra idea rectora es la siguiente, los procesos transferenciales se producen en el transcurso de fuertes vinculaciones como las que ocurren en la escuela entre el estudiante y su educador, de allí que no hablemos de transferencia propiamente dicha, sino de fenómenos transferenciales.

Es Janine Filloux quien desde 1989 publica en la Revista Francesa de Pedagogía un artículo donde propone a partir de textos dedicados al tema de "pedagogía psicoanalítica" la cuestión acerca de la transferencia, afirmando que el abordaje de los fenómenos transferenciales en la práctica pedagógica puede permitir aclarar la naturaleza de lo pedagógico.

En la situación clínica en "la teoría de la cura" las manifestaciones transferenciales se organizan en una neurosis artificial llamada neurosis de transferencia, estas se establecen primero como obstáculo para la cura, como resistencia del retorno de lo reprimido pero luego se convierte en el instrumento de la cura analítica a condición de interpretar con éxito su sentido para el "enfermo".

Así la situación de transferencia es una reviviscencia que reanima los deseos incestuosos de la primera infancia, y la infancia, la situación edípica en todas sus facetas. Pero tenemos que tomar en cuenta que, la noción de transferencia, convoca a la vez a la noción de contra-transferencia, la cual representa "el conjunto de reacciones inconscientes del analista en la persona del analizado (...)" (Laplanche y Pontalis, 1998, p.103)

Asumir el marco de referencia psicoanalítico tiene implicaciones para la concepción de la práctica pedagógica como ya lo hemos mencionado, en especial, el maestro tendrá que dejar de lado al "niño imaginario" de la pedagogía, nosotros en las aulas tenemos que vérnoslas no solo con procesos de conocimiento en abstracto, sino con una serie de simbolizaciones que los niños (as), adolescentes, familias y los mismos educadores construyen alrededor del proceso de aprendizaje.

Sabemos, por ejemplo, que rechazos al profesor se traducen a menudo en rechazos a la materia que imparte, lo que para algunos alumnos conduce con los años a tomar decisiones vocacionales donde ésta no esté incluida (Lafortune, L., 1997) 
Si nosotros como educadores y educadoras pudiéramos descifrar esta red simbólica e imaginaria que se construye en las aulas, podríamos crear, sin duda, mejores condiciones de aprendizaje, pues los alumnos no son solo intelecto, actitudes y aptitudes, también están constituidos por afectos inconscientes que se disparan en toda relación significativa y que inciden en el desempeño escolar.

Una `práctica educativa con orientación psicoanalítica implica la reflexión constante del educador, a cerca de su lugar en el proceso y sobre sus propias expectativas en torno a los niños y niñas del salón de clase. Esto permite que el educador identifique el nivel de actuación de la transferencia (y contra-transferencia). Luego son dos tareas las que debe realizar el educador; la primera se refiere a cómo manejar esta transferencia (y contratransferencia), la segunda, cómo provocar, instaurar y construir una transferencia positiva de parte del estudiante sobre él como educador (Filloux, 1989).

\subsubsection{Construir la transferencia}

Nos dice Filloux (1989) que instaurar la transferencia es un tema que nos reenvía a tomar en cuenta la necesidad de una alianza narcisística entre el alumno y el educador, allí donde esta no está instalada normalmente por el efecto mismo de la situación escolar.

De lo dicho se desprende que el educador deberá buscar esta posición de influencia sobre sus estudiantes de una manera activa. Lo cual no será difícil considerando que el niño no tiene aún un yo fuertemente establecido, lo cual lo hace más influenciable.

Al respecto queremos subrayar aquí los riesgos que se pueden generar debido al establecimiento de una relación entre educador y estudiante donde el primero sea sentido como un padre o madre. Esto puede desencadenar una serie de identificaciones o desplazamientos de conflictos psíquicos sobre el educador, quien debe ser percibido como un guía, como un mediador en el proceso.

Pero si el maestro logra ganarse, por así decir, la confianza del niño, que no se deba a una compensación narcisística mediante la cual el maestro-adulto obtenga como prioridad satisfacciones personales. El adulto deberá conservar su mirada en el horizonte del proceso y no en la inmediatez de la satisfacción de sentirse bueno y querido. 


\subsubsection{Cómo manejar la transferencia.}

Pero además de provocar o instaurar la transferencia en los jóvenes educandos, el educador también estará llamado a mantener el límite de esta transferencia, evitando los "desbordamientos afectivos". Esta es precisamente la cuestión que deberá hacer preguntar al docente por su contratransferencia para mantener un lazo estable y equilibrado.

De ninguna manera queremos decir que el docente deba sustituir su capacidad intuitiva y espontaneidad en el trabajo con niños, no se trata de obviar su preparación pedagógica a cambio de un saber psicoanalítico. A lo que nos conduce esta propuesta es a la disposición del educador para actuar y no reaccionar frente a los sentimientos que sus alumnos le provocan como la frustración o el enojo, el bienestar o el amor.

Lo que planteamos es la necesidad de que el educador se detenga un momento en estos procesos que aunque no se puede dar cuenta fácilmente de ellos se refieren a aspectos que tienen un papel fundamental en la construcción del conocimiento. $Y$ en la disponibilidad del docente para continuar realizando su labor de la manera más profesional posible.

Esto requiere un profesional con una importante maestría personal. Esto es una persona que reconoce sus deseos, sus carencias y sus fragilidades, que se permite reflexionar sobre su práctica y que permite la crítica positiva acerca de lo que hace. Un educador abierto al diálogo con sus estudiantes, con sus colegas y consigo mismo.

\section{Conclusiones}

Para nosotros son dos las conclusiones fundamentales que podemos extraer de esta reflexión al respecto de la relaciones transferenciales en el aula.

La primera nos remite a la elección del psicoanálisis como conjunto de conocimientos teóricos que pueden aclarar la práctica educativa, siendo evidente que no debe ser propuesto como teoría dogmática que legisla la práctica pedagógica.

Nuestra segunda conclusión se refiere al tipo de formación que requieren los educadores. Consideramos que debe garantizarse desde el currículo una sólida formación 
pedagógica que permita al educador conducir adecuadamente los procesos de aprendizaje en el salón de clase. Pero también queremos subrayar la necesidad de una sólida formación en el sentido de un acompañamiento y de una constante re-flexión acerca de los procesos personales que el estudiante de educación vivencia durante su formación académica, pero también durante su práctica pedagógica como profesional de la educación.

Aquí puede parecer que somos tentados a caer en una especie de idealismo acerca de la posibilidad de brindarle a estos profesionales espacios para el análisis de sus vivencias, no obstante, a partir de nuestra práctica, es una certeza la necesidad de que nuestros estudiantes universitarios tengan la oportunidad de realizar esta revisión personal sobre sus patrones de interacción y acerca de los ideales educativos con los que se enfrenta cotidianamente en su práctica profesional.

Para nosotros la teoría psicoanalítica es una fuente para iniciar este proceso de reflexión, cierto es que desde esta propuesta teórica los ideales quedan cuestionados pero ya lo hace la realidad misma. Por esto, invitamos a construir visiones a futuro más realistas, no por ello menos satisfactorias. El reconocimiento de la propia imposibilidad de conquistar el ideal educativo nos permite acercarnos con mayor claridad a la realidad del aula, a la realidad sobre nosotras mismas (os) y sobre nuestros estudiantes. Todo ello puede generar un aprendizaje más humanizado donde se permita el encuentro mutuo entre los que participan de este proceso al que llamamos educativo. 


\section{Referencias}

Assoun, P. (1997). Psychanalyse. Paris: Presses Universitaires de France.

Bowlby, J. (1989). Una base segura. Aplicaciones clínicas de una teoría del apego. Buenos Aires: Paidós.

Berk, L. (1999). Desarrollo emocional. Capítulo 10. En Desarrollo del niño y del adolescente. Madrid: Prentice Hall Iberia, p. 514-569.

Chevallard, Y. (2000). La transposición didáctica. Del saber sabio al saber enseñado. Buenos Aires: AIQUE.

Dengo, M.E. (1998). Educación Costarricense. San José : EUNED.

Filloux, J. (1989). Sur le concept de transfert dans le champ pédagogique. Revue Française de Pédagogie, 87, 59-75.

Freud, S. (1913-14/1997). Sobre la psicología del colegial (1914). Obras Completas. Buenos Aires: Amorrortu.

Freud, S. (1932-36/1979). Lecciones de introducción al psicoanálisis. Nuevas Conferencias de Introducción al Psicoanálisis y otras Obras. Buenos Aires: Amorrortu.

Freud, S. (1933/1996). Eclaircissement, applications, orientations, XXXIV. Nouvelles Conférences de Psychanalyse. Oeuvres Complètes. Paris: Folio Essais. Gallimard.

Freud, S. (1937/1981). Análisis terminable e interminable. Tomo III. Obras Completas. Madrid: Biblioteca Nueva.

Freud, S. (1933/1984). Sur une Weltanschauung, XXXV. Nouvelles Conférences de Psychanalyse. Paris: Folio Essais. Gallimard.

Green, A. (1973/1992). Le discours vivant. Paris: Presses Universitaires de France.

Hubert, R. (1965). Traité de pédagogie générale. Paris: Presses Universitaires de France.

Lafon, R. (1963). Vocabulaire de psychopédagogie et de psychiatrie de l'enfant. Paris: Presses Universitaire de France.

Lafortune, L. (1997). Dimension affective en mathématiques. Bruxelles: De Boeck.

Laplanche, J. y Pontalis, J-B. (1998/1963). Vocabulaire de la psychanalyse. Paris: Presses Universitaires de France.

Lerbet, G. (1980). L'Archeo-Pedagogie. Essai d'analyse structuraliste de la genèse du concept pédagogique. Revue Française de Pédagogie, 52, 4-18.

Moll, Jeanne (1989). La pédagogie psychanalytique. Origine et histoire. Paris: Dunod. 
Mosconi, N. (1986). De l'application de la psychanalyse à l'éducation. Revue Française de Pédagogie, 75, 73-79.

Sotelo, I., (1999). Educación y Democracia. En Volver a pensar la educación. (Vol.1). Madrid : Ediciones Morata, S.L.

Steichen, R., (1997). L'Identité du sujet: sa construction et ses nominations. En Identification et identité dans les familles individu? Personne? Sujet? Bruxelles: Academia Bruylant, p.11-46. 